J. Phys. IV France 138 (2006) 203-212

(C) EDP Sciences, Les Ulis

DOI: $10.1051 /$ jp4:2006138023

\title{
Dopage laser en microélectronique
}

\author{
T. Sarnet ${ }^{1}$, M. Hernandez ${ }^{2}$, D. Débarre ${ }^{1}$ et J. Boulmer ${ }^{1}$ \\ ${ }^{1}$ Institut d'Électronique Fondamentale, UMR CNRS 8622, Université Paris-Sud, \\ Bât. 220, Centre Scientifique d'Orsay, 91405 Orsay Cedex, France \\ 2 SOPRA-SA, 26 rue Pierre Joigneaux, 92270 Bois-Colombes, France
}

\begin{abstract}
Résumé. Les procédés de recuit et de dopage laser du silicium ont été étudiés de manière intensive au cours des précédentes décennies. Ces études ont d'ores et déjà permis un transfert technologique pour la fabrication des semiconducteurs. Cependant les futures générations CMOS vont nécessiter des techniques de dopage encore plus fines, capable de fabriquer les jonctions ultra-minces décrites dans la "roadmap" de l'ITRS (International Technology Roadmap for Semiconductors). Ce papier décrit des résultats récents obtenus dans le cadre du RMNT DOLAMI consacré au dopage laser de jonctions ultra-minces, ainsi que des applications du dopage laser pour les microtechnologies liées à la fabrication de MEMS.
\end{abstract}

\section{INTRODUCTION}

Parmi les techniques susceptibles de réaliser les jonctions ultra-minces de la microélectronique émergent deux procédés laser : le LTP et le GILD. Ces procédés laser sont basés sur des cycles de chauffage/refroidissement du substrat, avec ou sans phase de fusion du matériau. La fusion superficielle du matériau permet l'épitaxie en phase liquide du silicium cristallin sous-jacent pendant la solidification. Dans le cas du LTP les dopants sont implantés préalablement, alors que dans le GILD les dopants sont incorporés directement pendant le traitement laser [1-12].

Les différents paramètres de l'interaction (longueur d'onde, densité d'énergie ou de puissance, durée d'impulsion...) changent de manière considérable la façon dont le matériau se comporte (absorption, diffraction...) et par conséquent les caractéristiques finales des jonctions obtenues (concentration en dopant, forme du profil de dopage, résistivité...).

Des résultats récents obtenus par des laboratoires de recherche académique (Stanford Univ., CNR Rome, Univ.of Oslo, IEF Orsay etc...) et des industriels des semiconducteurs et fabricants de laser (Applied Materials, Ultratech steppers, Lambda Physik, SOPRA SA...) ont démontré tout l'intérêt des procédés laser pour l'activation des futures jonctions ultra-minces (technologies $22-32 \mathrm{~nm}$ ). Les résultats obtenus sont supérieurs aux performances des meilleures technologies actuelles.

D'autres applications du dopage laser sont aussi présentées comme la fabrication de micro et nanorésonateurs, de plus en plus utilisés dans l'industrie des micromécanismes de type MEMS.

\section{DOPAGE LASER POUR TRANSISTORS CMOS}

L'enjeu de cette étude est de montrer l'intégration du procédé et la faisabilité de cette technique pour la réalisation de transistors CMOS. A ce haut niveau d'intégration, l'influence de la distribution et de la taille des structures sur la diffraction du faisceau incident doit être prise en compte. Des simulations FEMLAB mettent en évidence l'importance de l'influence des longueurs de grille et de leurs espacements sur le couplage de la lumière laser avec les structures. La figure 1 est une cartographie du champ E en polarisation TE qui montre la diffraction de l'onde incidente causée par les structures de grille en poly-silicium avec des espaceurs (spacers) en nitrure de silicium et des diélectriques en oxyde de silicium.

En multipliant la partie imaginaire de l'indice optique des différents matériaux par le carré du champ E, on obtient une cartographie de l'absorption au niveau du matériau (Fig. 2). On voit que l'absorption 


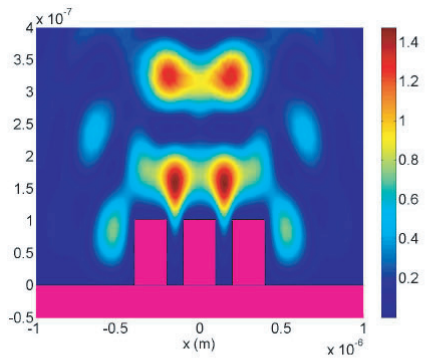

(a)

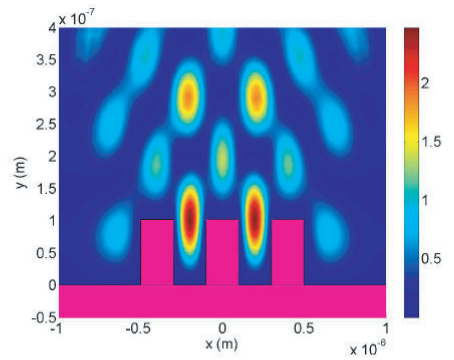

(b)

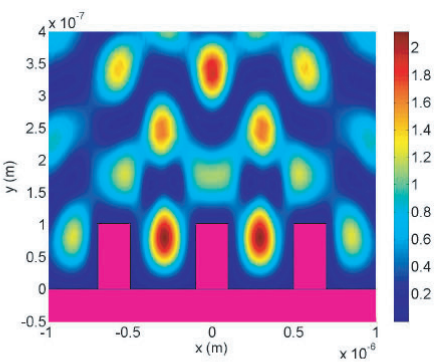

(c)

Figure 1. Cartes du champ E en polarisation TE pour trois grilles de longueur $200 \mathrm{~nm}$ et des espacements de 100 $\mathrm{nm}$ (a), $200 \mathrm{~nm}$ (b) et $400 \mathrm{~nm}$ (c). La hauteur de la grille est de $100 \mathrm{~nm}$.

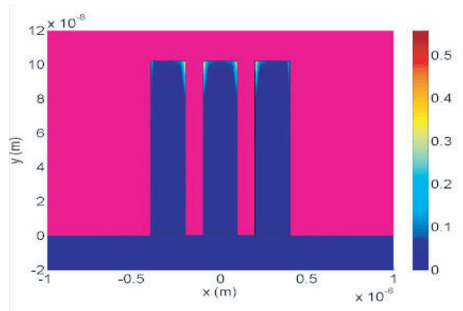

(a)

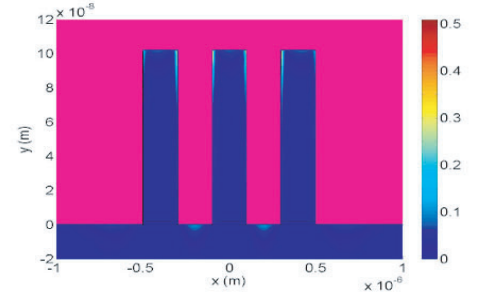

(b)

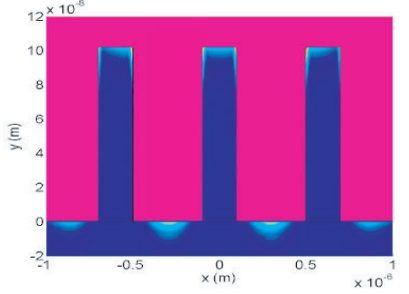

(c)

Figure 2. Cartes du champ absorbé en polarisation TE pour trois grilles de longueur $200 \mathrm{~nm}$ et des espacements de ea $100 \mathrm{~nm}$ (a), $200 \mathrm{~nm}$ (b) et $400 \mathrm{~nm}$ (c). La hauteur de la grille est de $100 \mathrm{~nm}$.

du rayonnement est fortement limitée entre les grilles (Fig. 2a) lorsque la distance inter-grille est très faible (100 $\mathrm{nm}$ pour une hauteur de grille de $100 \mathrm{~nm}$ également), on note aussi que la partie supérieure des grilles a tendance à absorber d'avantage de rayonnement.

La possibilité d'utiliser des revêtements multicouches optiques (silices, nitrures...) ou thermiques (couche absorbante) a donc été envisagée dans cette étude. En effet, le couplage de l'énergie laser dans la grille et dans les zones de S/D peut être amélioré en utilisant des couches supplémentaires, réfléchissantes et anti-réfléchissantes convenablement choisies. Une des solutions consiste à protéger la grille du transistor en déposant un matériau qui va augmenter la réflexion sur la grille, et donc diminuer l'énergie absorbée par la grille [3]. Le schéma de principe utilisé est représenté sur la figure 3. Pour ces tests, nous avons utilisé une plaque réalisée par lithographie e-beam, munie d'un empilement nitrure/oxyde de silicium c'est-à-dire : $\mathrm{Si}_{3} \mathrm{~N}_{4}(20 \mathrm{~nm}) / \mathrm{SiO}_{2}(60 \mathrm{~nm}) / \mathrm{grille}$ et $\mathrm{Si}_{3} \mathrm{~N}_{4}(20 \mathrm{~nm}) / \mathrm{SiO}_{2}(10 \mathrm{~nm}) /$ Silicium. Les échantillons ont été préalablement implantés au bore $(\mathrm{B}+, 3$ $\mathrm{keV}, 3.10^{15} \mathrm{at} / \mathrm{cm}^{2}$ ) pour réaliser les extensions source et drain.

Les sources laser utilisées sont le VEL SOPRA (15 Joules, $200 \mathrm{~ns}, 308 \mathrm{~nm}$ ) et un Lambda Physik Compex 102 (200 mJ, $28 \mathrm{~ns}, 308 \mathrm{~nm}$ ), avec leurs systèmes d'homogénéisation de faisceau basés sur des matrices de micro-lentilles. Ces systèmes permettent une homogénéisation du faisceau incident de $1 \%$ et 5\% respectivement pour le laser VEL SOPRA et le Lambda Physik Compex 102.

La figure 4 montre les profils SIMS des échantillons avec $10 \mathrm{~nm}$ de silice et 20, 25 et $30 \mathrm{~nm}$ de nitrure de silicium. Ils montrent que, pour obtenir une même profondeur fondue, il est nécessaire d' augmenter la densité d'énergie : $1,2,1,4$ et $1,8 \mathrm{~J} / \mathrm{cm}^{2}$ pour 20,25 et $30 \mathrm{~nm}$ de $\mathrm{Si}_{3} \mathrm{~N}_{4}$ respectivement. La figure 4 montre également un profil SIMS obtenu sur du silicium nu : l'épaisseur de jonction est bien plus faible pour 


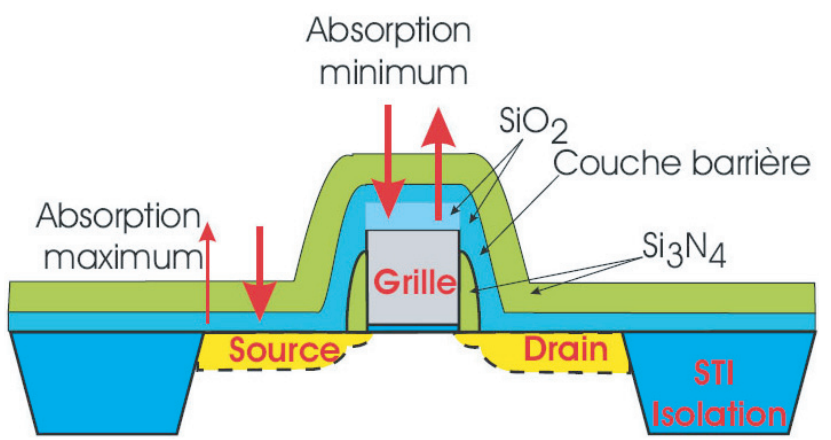

Figure 3. Principe de la structure utilisée pour obtenir des couches réfléchissantes et anti-réfléchissantes utilisant des empilements $\mathrm{Si} 3 \mathrm{~N} 4 / \mathrm{SiO} 2 / \mathrm{Si}$.

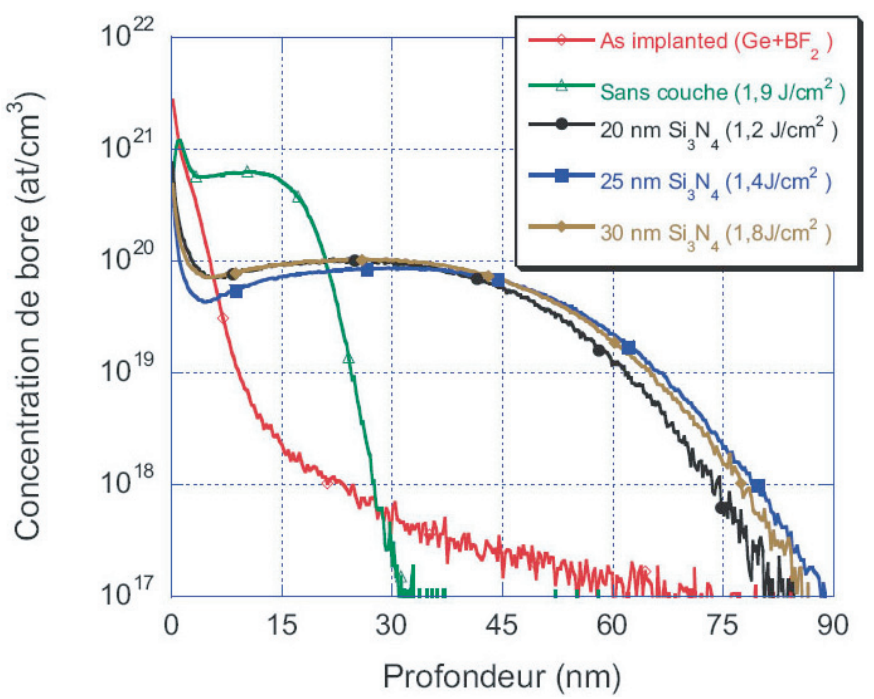

Figure 4. Profils SIMS obtenus après recuit laser sur un substrat avec différentes couches de nitrure déposées sur un substrat avec $10 \mathrm{~nm}$ d'oxyde de silicium préalable (laser SOPRA).

une densité d'énergie pourtant plus élevée $\left(1,9 \mathrm{~J} / \mathrm{cm}^{2}\right)$ ce qui confirme encore l'efficacité des couches $\mathrm{Si}_{3} \mathrm{~N}_{4} / \mathrm{SiO}_{2}$ anti-réfléchissantes sur le procédé de recuit.

Les dépôts ont été réalisés à haute température $\left(>750^{\circ} \mathrm{C}\right)$. L'utilisation de la préamorphisation pour la source et le drain est donc exclue car les jonctions seraient recristallisées lors du dépôt des couches d'oxyde et de nitrure. Cependant ce type d'empilement est intéressant car il permet un meilleur couplage entre l'irradiation laser et les structures.

Les longueurs de grille des transistors testés vont de $10 \mu \mathrm{m}$ à $40 \mathrm{~nm}$ et leurs largeurs sont de $10 \mu \mathrm{m}$.

Compte tenu des tests effectués précédemment, nous avons estimé que le seuil de fusion se situait vers $0,9 \mathrm{~J} / \mathrm{cm}^{2}$, pour les régions de source et drain, et au delà de $3 \mathrm{~J} / \mathrm{cm}^{2}$ pour la grille (cf. Fig. 5). Les densités d'énergie laser ont été variées de 0,25 à $1 \mathrm{~J} / \mathrm{cm}^{2}$, c'est-à-dire dans des conditions où le LTP est en régime de "sub-melt" et proche du seuil de fusion des source et drain.

Dans un premier temps, nous avons vérifié les caractéristiques obtenues par un recuit classique RTP. Ces mesures ont pour but de confirmer que toutes les étapes technologiques se sont bien déroulées et ont permis d'obtenir des transistors fonctionnels avec un recuit classique. Sur la figure 6 sont représentées 


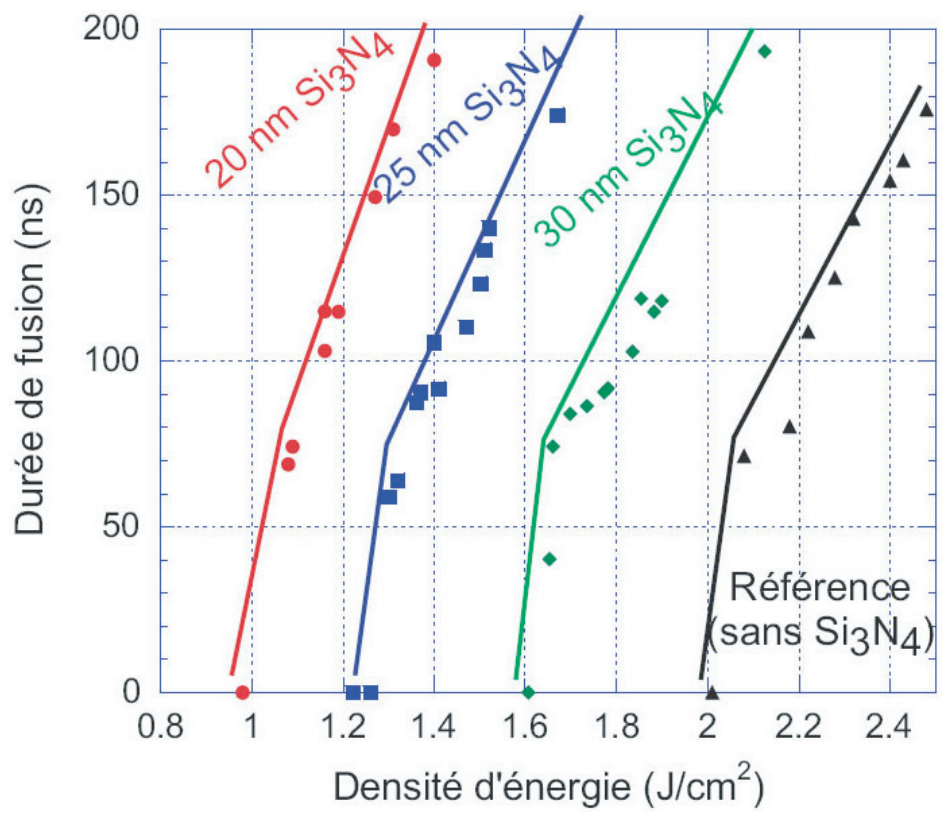

Figure 5. Influence de l'épaisseur de nitrure déposé sur $10 \mathrm{~nm}$ d'oxyde de silicium sur l'évolution de la durée de fusion (laser SOPRA).

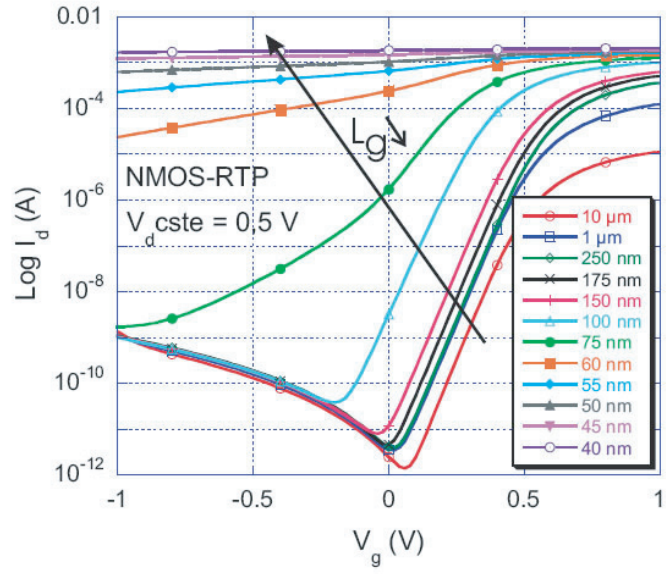

(a)

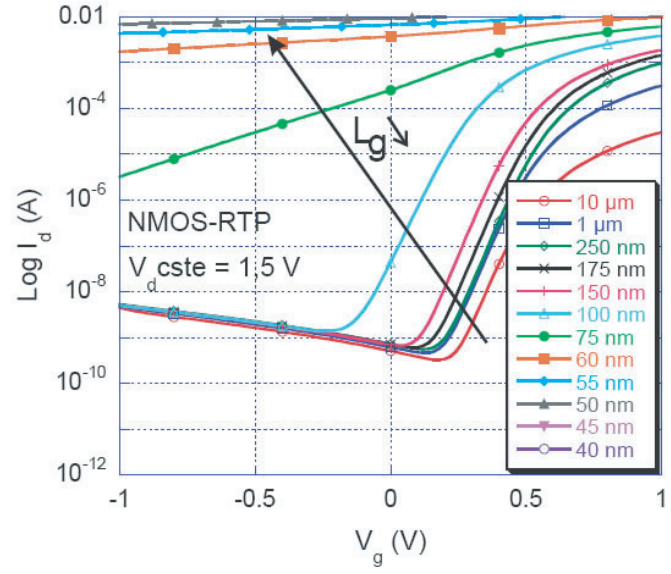

(b)

Figure 6. Caractéristiques courant de drain (Id) et fonction de la tension de commande (Vg) pour Vd faible $(0,5 \mathrm{~V})$ (cas a) et $\mathrm{Vd}$ fort $(1,5 \mathrm{~V})$ pour des transistors dont les longueurs de grilles varies de $10 \mu \mathrm{m}$ 'a $40 \mathrm{~nm}$.

les caractéristiques Id en fonction de $\mathrm{Vg}$ à Vd faible $(0,5 \mathrm{~V})$ (figure 6a) et Vd fort (1,5 V) (Fig. 6b). On observe que les transistors ne sont plus fonctionnels pour des longueurs de grille inférieures à $75 \mathrm{~nm}$.

Nous avons par la suite comparé ces résultats à ceux obtenus par LTP. La figure 7 présente les caractéristiques de fonctionnement $\mathrm{I}_{\text {off }}$ en fonction de $\mathrm{I}_{\text {on }}$ de transistors PMOS ayant été recuits par RTP (points noirs) ou par LTP (points verts). Chaque groupe de points correspond à une série de résultats de mesures effectuées sur différents transistors ayant la même largeur de grille. On constate tout d'abord 


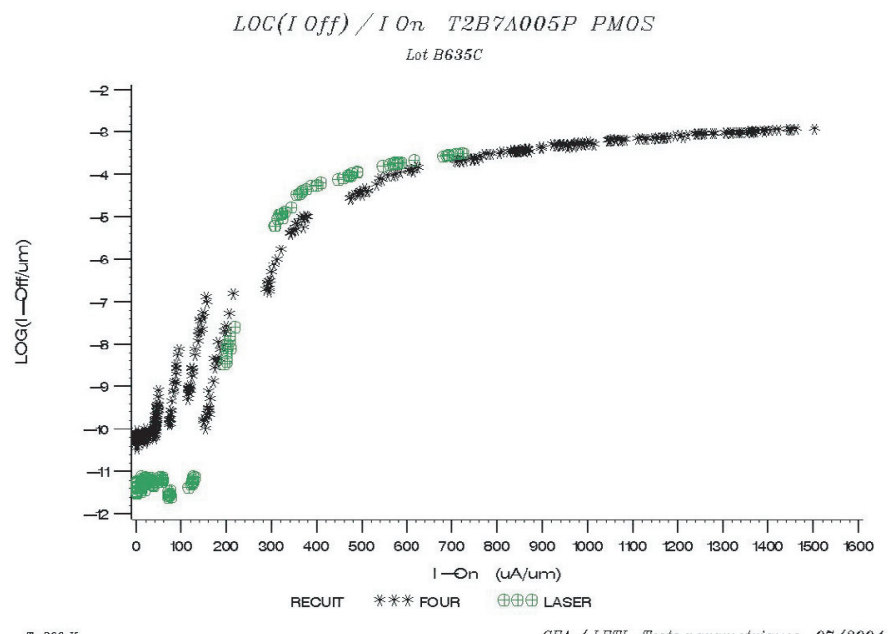

Figure 7. Caractéristiques Ion-Ioff des transistors PMOS après recuit laser (points verts) et recuit RTP (points noirs).

que les transistors réalisés avec cette technologie ne sont réellement fonctionnels que pour des longueurs de grille supérieures ou égales à $75 \mathrm{~nm}$, quelque soit la technique de recuit. Les résultats obtenus avec les transistors PMOS (dopage au bore) sont encourageants. En effet, les résultats montrent (Fig. 7) que, pour des longueurs de grille inférieures ou égales à $75 \mathrm{~nm}$, leurs caractéristiques $\log \left(\mathrm{I}_{\mathrm{off}}\right) / \mathrm{I}_{\mathrm{on}}$, sont meilleures avec un recuit LTP qu'avec un recuit classique. En dessous de $75 \mathrm{~nm}$, aucune des deux techniques de recuit n'est satisfaisante. Remarquons que tous les points correspondant au recuit laser ont été obtenus avec des densités d'énergie allant de 0,25 à $1 \mathrm{~J} / \mathrm{cm}^{2}$.

La variation de la densité d'énergie laser semble donc avoir un effet mineur : il est probable que les températures élevées atteintes lors des dépôts d'oxyde HTO et de nitrure sont responsables d'un recuit au moins partiel des couches implantées. Quoiqu'il en soit, des expériences complémentaires seront nécessaires pour pouvoir conclure définitivement. Les premiers résultats obtenus avec ce lot électrique sont prometteurs, car ils démontrent que le recuit laser peut être utilisé pour remplacer l'étape de recuit RTP. Il est effectivement possible de réaliser des transistors fonctionnels, notamment en utilisant les deux couches d'oxyde et de nitrure. Il subsiste cependant des points à éclaircir et de nouveaux essais sont à réaliser afin d'optimiser les conditions d'implantation, les épaisseurs et la nature des couches encapsulantes et les conditions laser.

Chacune des jonctions réalisées a été caractérisée in situ en temps réel par réflectivité transitoire et, après traitement par de nombreuses techniques telles que : SIMS, SRP, mesure 4 pointes, ellipsométrie, diffraction par rayons X, AFM, MEB et TEM.

La figure 8 permet de comparer les résistivités surfaciques et les profondeurs de jonction obtenues par recuit laser au cours de nos expériences à celles obtenues par d'autres techniques tel que les recuits "spike-RTP", "SPER", lampes Flash ou laser (les rectangles représentent les spécifications de l'ITRS [14]).

Les observations MEB de révélations chimiques TMAH ont permis de montrer qu'il n'y avait pas :

- de détérioration de la grille et de l'oxyde de grille,

- de diffusion latérale sous la grille de la zone fondue et que la zone de dopants activés restait confinée à la zone préamorphisée [10-11].

Des mesures AFM ont montré que des rugosité RMS voisines de $1 \AA ̊$ peuvent être atteintes après recuit laser pourvu que l'épitaxie en phase liquide puisse avoir lieu à partir du silicium monocristallin sous-jacent. Pour des densités d'énergie proche du seuil de fusion, le front de fusion peut ne pas se 


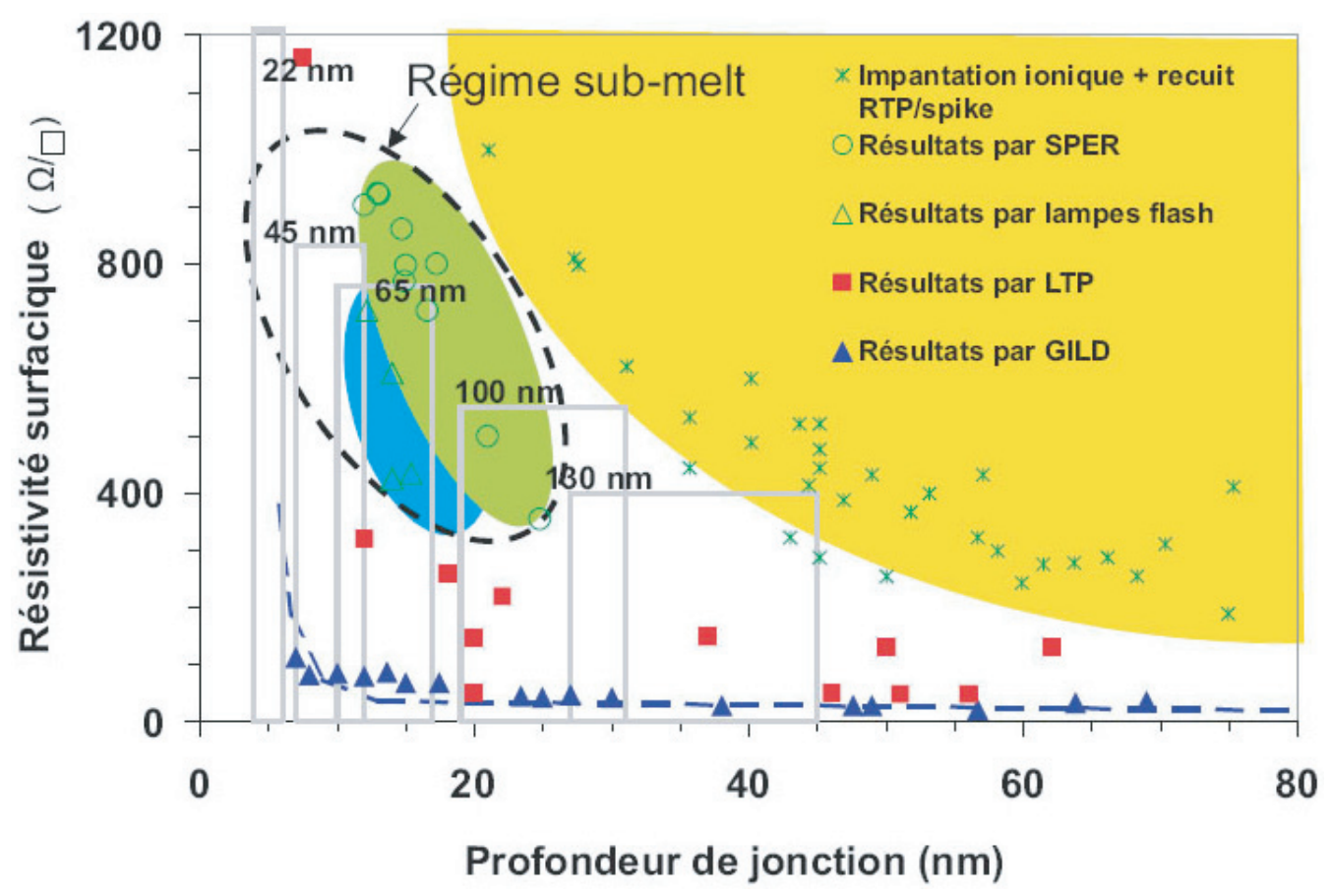

Figure 8. Résistivités surfaciques en fonction de la profondeur de jonction obtenues par différentes techniques de recuit classiques [15], avancées [16] et laser et comparées aux spécifications de l'ITRS [14] pour les futures générations de la technologie CMOS (rectangles).

propager jusqu'au Si-c : on observe alors une augmentation de rugosité, cette rugosité RMS reste cependant inférieure à $2 \mathrm{~nm}$. La figure 9 montre un cliché TEM de la structure cristallographique obtenue après un recuit laser: on note l'absence de défauts dans la zone recuite (épaisseur $20 \mathrm{~nm}$ ), l'épaisseur d'oxyde est de $4 \mathrm{~nm}$ et la rugosité RMS de l'ordre de $2 \AA$.

\section{DOPAGE LASER POUR LES MEMS}

La bonne qualité des jonctions obtenues permet d'envisager d'utiliser ces procédés laser pour réaliser des étapes technologiques de salle blanche, pour la fabrication de MEMS par exemple. La figure 10 montre des structures vibrantes suspendues réalisées par dopage laser direct (GILD) sur du silicium (100). Les masques durs ont été gravés par lithographie électronique sur de la silice. Le dopage laser a donc été réalisé de manière sélective à travers ces masques (dopage au bore), puis après suppression du masque de silice les échantillons ont subi une attaque chimique anisotrope (TMAH) permettant de libérer les structures. La combinaison de ces différentes étapes technologiques permet ainsi d'envisager la fabrication de structures aux dimensions très réduites ( $100 \mathrm{~nm}$ et moins) sur du silicium monocristallin pour la réalisation de MEMS [11-13].

Afin de caractériser in-situ la structure des ponts par diffraction $X$ une source micro faisceau de rayons $\mathrm{X}$ (rayonnement synchrotron de troisième génération, ligne $\mu$ - Diffraction 7.3.3 de l'Advanced Light Source (ALS) du Lawrence Berkeley National Laboratory, USA) a pu être utilisé: les résultats préliminaires montrent la faisabilité de la méthode de caractérisation ainsi que les décalages des paramètres de maille de la structure du silicium (jusqu'à $2,8 \%$ ) induits par la forte concentration en bore [13]. Ces fortes contraintes permettent d'augmenter considérablement les fréquences de résonance pour les structures MEMS, il a par ailleurs été observé optiquement (laser doppler) des augmentations d'un facteur 25 de ces fréquences par rapport au silicium non dopé [12]. 


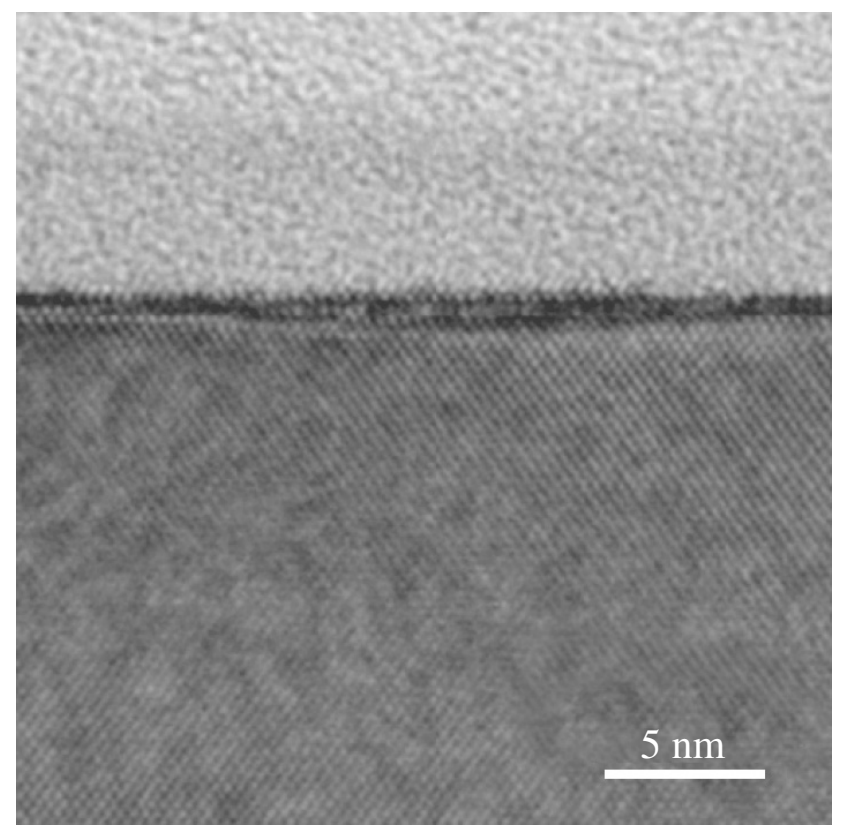

Figure 9. Observation par microscopie électronique en transmission en coupe sur un échantillon préamorphisé $\left(\mathrm{Ge}+, 10 \mathrm{keV}, 10^{15} \mathrm{at} / \mathrm{cm}^{2}\right)$, implanté au $\mathrm{BF}^{+}\left(2,2 \mathrm{keV}, 2.10^{15} \mathrm{at} / \mathrm{cm}^{2}\right)$ puis recuit à une densité d'énergie de 2,20 $\mathrm{J} / \mathrm{cm}^{2}$ (laser SOPRA).
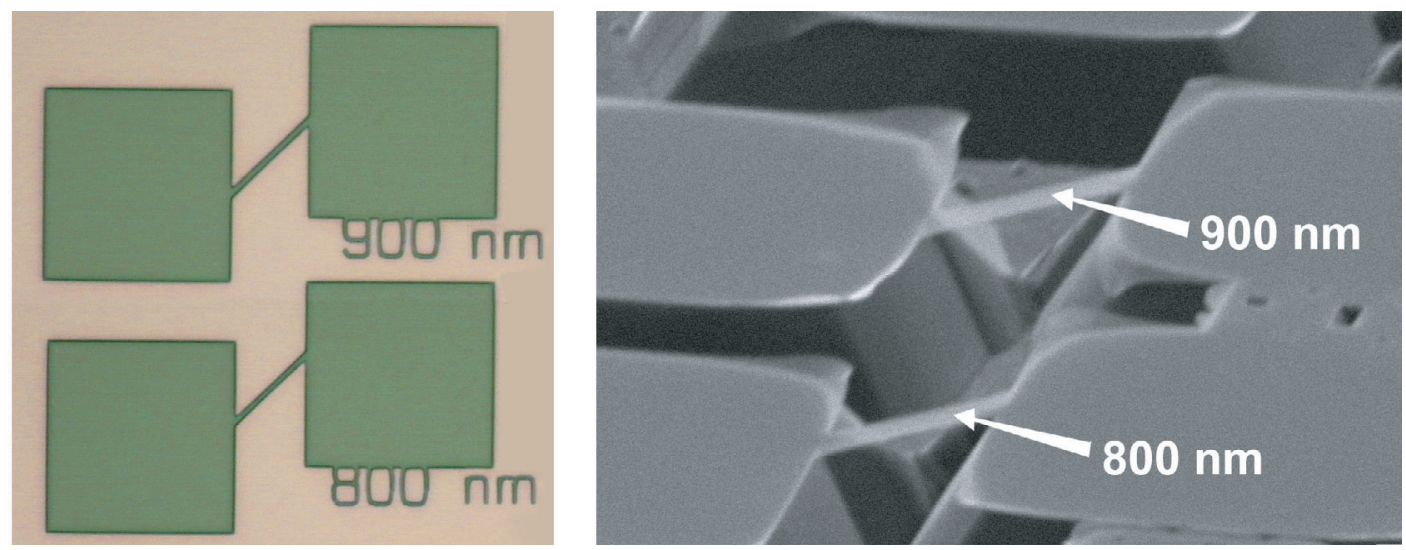

Figure 10. Réalisation de structures vibrantes (ponts) par dopage laser direct (GILD) pour l'industrie des MEMS. Gauche: vue de dessus avant dopage, la zone rose (clair) représente le masque dur de silice, la zone verte (plus foncée) est le silicium nu à doper. Droite: vue MEB après dopage et libération des structures au TMAH. Dimensions des ponts : longueur $10 \mu \mathrm{m}$, largeur 800 et $900 \mathrm{~nm}$, épaisseur $100 \mathrm{~nm}$.

\section{DISCUSSION}

La réalisation de jonctions ultra-minces et fortement dopées est un enjeu majeur pour la poursuite de la miniaturisation des dispositifs de la microélectronique et de son composant de base, le transistor MOS. Actuellement aucune technique testée, associant des implantations à très faible énergie et des recuits de plus en plus rapide ne répond aux exigences de l'ITRS pour les noeuds technologiques sub-60 nm. La technique de recristallisation en phase solide (SPER) permet d'obtenir des jonctions très fines mais 
laisse encore des défauts dans le silicium, induisant de forts courants de fuite. Les autres techniques évaluées aujourd'hui par les fabricants de composants microélectronique, comme le recuit par lampes flash ou le balayage par faisceau laser continu, ne proposent des solutions industrielles que pour le noeud $65 \mathrm{~nm}$. Au cours de ce travail, nous avons montré que les techniques de recuit et de dopage par laser à excimères étaient bien adaptées à la réalisation de jonctions ultra-minces et pouvaient répondre aux exigences de l'ITRS pour tous les noeuds technologiques utilisant le silicium y compris pour les noeuds technologiques $45 \mathrm{~nm}$ et inférieurs.

En parallèle de toutes les expériences de recuit laser effectuées à l'IEF et à SOPRA, de nombreux calculs de simulation thermique ont été réalisés et nous ont permis de disposer d'un outil d'évaluation du procédé laser indispensable. Nous avons évalué des rampes de montée en température de $10^{10} \mathrm{~K} / \mathrm{s}$ qui se situent plusieurs ordres de grandeur au dessus des celles obtenues par les lampes flash ou les lasers continu CW. Les profondeurs fondues ont pu aussi être prédites et les densités d'énergie laser optimisées.

Les expériences de recuit laser sur les dispositifs expérimentaux à partir de nombreuses plaques de $200 \mathrm{~mm}$ non structurées, et les nombreuses caractérisations réalisées au LETI, à SOPRA et à l'IEF, ont permis de quantifier les effets de la durée de l'impulsion des lasers utilisés et ont défini les différentes fenêtres de procédé explorables pour de futures irradiations sur dispositifs.

Le mode "sub-melt", c'est-à-dire sans fusion a aussi été étudié: il représente une solution à court terme pour obtenir des jonctions activées par des recuits laser bien plus rapides que les recuits classiques actuellement utilisés, à condition que les jonctions n'aient pas subi de préamorphisation.

Les caractérisations de profils d'activation par SIMS et SRP ont montré que les deux lasers utilisés permettaient d'obtenir des profils de bore carrés et extrêmement abrupts $(2 \mathrm{~nm} /$ décade). Les caractérisations par TEM et diffraction par rayons $\mathrm{X}$ ont souligné la très bonne qualité cristalline des couches recuites par LTP ou dopées par GILD.

Dans le cas du dopage GILD une incorporation record a été atteinte avec près de $10^{14}$ atomes de bore $/ \mathrm{cm}^{2}$ et par tir laser ce qui a permis d'obtenir des concentrations en bore supérieures à $10^{21}$ atomes de bore $/ \mathrm{cm}^{3}$.

Une collaboration avec la société IBS, spécialiste de l'implantation ionique qui développe un outil industriel d'implantation par immersion plasma, nous a fait bénéficier de substrats implantés à très faible énergie où les profils d'implantation sont inférieurs à $10 \mathrm{~nm}$. Cette étude nous a permis de vérifier que, grâce à l'impulsion laser de $200 \mathrm{~ns}$, nous pouvions obtenir des jonctions ayant des résistivités surfaciques environ cinq fois plus faibles que par RTP.

Les premiers résultats électriques obtenus sur ces dispositifs sont très encourageants pour les techniques de dopage par laser puisqu'ils montrent que ce procédé de recuit permettait d'obtenir des transistors CMOS fonctionnels. En effet, l'enjeu majeur de cette technique est son intégration au sein des procédés actuels de réalisation, avec un minimum d'étapes supplémentaires.

Les résultats obtenus au cours de cette étude permettent d'envisager les suites à court terme de ce travail, notamment la vérification et l'optimisation des résultats électriques obtenus en testant de nouveaux empilements de couches réfléchissantes et anti-réfléchissantes.

Ces empilements seront optimisés grâce aux calculs de simulation de diffraction électromagnétique par les structures afin d'obtenir un procédé laser pertinent pour tous les espacements existants entre les différentes grilles d'une même puce.

Les futures possibilités de procédés offertes par le recuit ultra-rapide LTP ou le dopage GILD pour l'industrie des semi-conducteurs sont nombreuses.

Citons :

- l'utilisation de substrats SOI qui permet de faciliter le couplage thermique entre le laser et le substrat.

- L'étude de l'association du recuit laser à d'autres techniques de recuit plus conventionnelles comme les recuits SPER ou le Spike-RTP. 
- l'emploi de nouveaux matériaux tels que des couches à forte permittivité $\left(\mathrm{HfO}_{2}\right)$, du silicium contraint ou du SOI ultra-mince.

- certains résultats préliminaires de simulations de diffraction électromagnétique qui ont montré que les effets de confinement de l'énergie laser pourraient être mis à profit au sein de structures constituées de plusieurs grilles ou FIN-FET.

- l'industrie des MEMS

\section{CONCLUSION}

Grâce au traitement laser nous avons pu obtenir des transistors PMOS fonctionnels, en utilisant des empilements de $\mathrm{SiO}_{2} / \mathrm{Si}_{3} \mathrm{~N}_{4}$, dont les caractéristiques sont meilleures après recuit laser qu'après recuit RTP, pour les nœuds supérieurs à $50 \mathrm{~nm}$. Par contre, les résultats préliminaires obtenus pour les transistors NMOS ne sont pas encore convaincants et nécessitent une étude plus approfondie afin de comprendre pourquoi les transistors dopés avec de l'arsenic ont des caractéristiques moins bonnes que ceux dopés au bore. L'objectif de cette étude a toutefois été atteint : confirmer l'intégration du procédé laser à excimère dans la chaîne de fabrication d'un transistor MOS.

Ces travaux montrent aussi la possibilité d'utiliser ces traitements laser pour la réalisation de structures suspendues pour l'industrie des MEMS. Les propriétés du silicium extrêmement dopé au bore par GILD, peuvent être exploitées afin d'augmenter considérablement les fréquences de résonance des structures tout en réduisant leur taille.

Les futurs travaux porteront sur des structures MOSFET utilisant d'autres empilements de couches optiques dont les épaisseurs auront été optimisées par des calculs de diffraction électromagnétique. Les études intégreront des structures proches de celles envisagées pour les futures générations de composants: substrats de SOI, utilisation de couches diélectriques "high-k", implantations très basse énergie, grilles métalliques, etc.

\section{Remerciements}

Une partie de ces travaux a été réalisée dans le cadre du programme national RMNT DOLAMI (DOpage LAser pour les jonctions ultra-MInces)). Les auteurs tiennent à remercier tous les participants du RMNT DOLAMI en particulier Cyrille Laviron, Marie-Noëlle Semeria, Denis Camel et J.-L. Santailler du CEA-LETI, D. Zahorski, Julien Venturini, Dominique Bérard de SOPRA-SA, Hassan Akhouayri et Franck Billard de l'Institut Fresnel de Marseille, ainsi que les participants au programme européen FLASH (Fundamentals and Applications of Laser Processing for Highly Innovative MOS Technology) pour les échanges de résultats et les discussions fructueuses.

Les auteurs remercient également Kuniyuki Kakushima de l'Université de Tokyo, pour son aide précieuse dans l'élaboration des structures MEMS ainsi que Frank Torregrossa de la société IBS pour les implantations par immersion plasma.

\section{Références}

[1] M. O. Thompson, J. W. Mayer, A. G. Cullis, H. C. Webber, N. G. Chew, J. M. Poate, and D. C. Jacobson, "Silicon melt, regrowth, and amorphisation velocities during pulsed laser irradiation", Phys. Rev. Lett., 50(12) (1983) 896.

[2] E. Fogarassy, C. Fuchs, S. De Unamuno, and P. Siffert, "Excimer laser induced melting of heavily doped silicon : a contribution to the optimization of the laser doping process", App. Surf. Science, 43 (1989) 316.

[3] H. Tsukamoto, H. Yamamoto, T. Noguchi, and T. Suzuki. Ultrashallow junctions formed by excimer laser annealing. Jpn. J. Appl. Phys., 32 (1993) 967. 
[4] K. A. Gable, K. Jones, M. E. Law, L. S. Robertson, and S. Talwar, "Electrical and structural characterization of boron implanted silicon following laser thermal processing", Mat. Res. Soc., 717 (2002) C1.10.1.

[5] G. Fortunato, L. Mariucci, M. Stanizzi, V. Privitera, C. Spinella, S. Coffa, E. Napolitani, "Fabrication of ultra-shallow junctions with high electrical activation by excimer laser annealing", Materials Science in Semiconductor Processing 4/5, (2001) 417.

[6] V. Privitera, C. Spinella, G. Fortunato, L. Mariucci, “Two-dimensional delineation of ultra shallow junctions obtained by ion implantation and excimer laser annealing”, Appl. Phys. Lett. 77, 552 (2000).

[7] A. La Magna, P. Alippi, V. Privitera, G. Fortunato, M. Camalleri, and B. Svensson, "A phase-field approach to the simulation of the excimer laser annealing process in si", J. Appl. Phys, 95(9) (2004) 4806.

[8] G. Kerrien, T. Sarnet, D. Débarre, J. Boulmer, M. Hernandez, C. Laviron and M. -N. Semeria, "Gas immersion laser doping (GILD) for ultra-shallow junction formation" Thin Solid Films, 453-454, (2004) 106-109.

[9] J. Venturini, M. Hernandez, G. Kerrien, C. Laviron, D. Camel, J. L. Santailler, T. Sarnet and J. Boulmer, "Excimer laser thermal processing of ultra-shallow junction: laser pulse duration" Thin Solid Films, 453-454 (2004) 145-149.

[10] M. Hernandez, J. Venturini, D. Berard, G. Kerrien, T. Sarnet, D. Débarre, J. Boulmer, C. Laviron, D. Camel, J.-L. Santailler, and H. Akhouayri, "Laser Thermal Processing using an optical coating for ultra shallow junction formation" Mat. Sci. and Eng. B, 114-115 (2004) 105-108.

[11] T. Sarnet, G. Kerrien, N. Yaakoubi, A. Bosseboeuf, E. Dufour-Gergam, D. Debarre, J. Boulmer, K. Kakushima, C. Laviron, M. Hernandez, G. Venturini, and T. Bourouina, "Laser doping for microelectronics and microtechnology" Appl. Surf. Sci. (2005), Special issue : Laser interactions in Materials : Nanoscale to Mesoscale, Elsevier Sciences Editor.

[12] Kuniyuki Kakushima, Thierry Sarnet, Gurwan Kerrien, Masanori Nagashio, Dominique Debarre, Jacques Boulmer, Takashi Noguchi, Tarik Bourouina, Hideki Kawakatsu, and Hiroyuki Fujita, Fabrication of Heavily Boron Doped Mechanical Resonators, 12th International Conference on Solid-State Sensors and Actuators, Transducers'03, 8-13 Jun. (2003) Boston, USA.

[13] P. Goudeau, N. Tamura, B. Lavelle, S. Rigo, T. Masri, A. Bosseboeuf, T. Sarnet, J. A. Petit, and J. M. Desmarres "X-Ray diffraction characterization of suspended structures for MEMS applications" Thin Films Stresses And Mechanical Properties XI 875 (2005) 121-126.

[14] The International Technology Roadmap for Semiconductor, 2003, http://public.itrs.net.

[15] D. Lenoble, A. Halimaoui, O. Kermarrec, Y. Campidelli, D. Bensahel, J. Bonnouvrier, O. Menut, E. Robilliart, E. Perrin, F. Arnaud, F. Boeuf, T. Skotnicki, A. Grouillet, and G. Bignell, "Integration of ultra-shallow junctions in sub-0.1 $\mu \mathrm{m}$ cmos transistors : What kind of process for a "safe" advanced technology ? International Workshop on Junction Technology, (2001) page 36.

[16] P.J. Timans, W. Lerch, S. Paul, J. Niess, T. Huelsmann, and P. Schmid "Usj formation : Annealing beyond 90nm" (2004) Solid State Technology. 